\title{
Three-dimensional Imaging Reveals New Compartments and Structural Adaptations in Odontoblasts
}

Journal of Dental Research 2015, Vol. 94(7) 945-954

(c) International \& American Associations for Dental Research 2015 Reprints and permissions: sagepub.com/journalsPermissions.nav DOI: 10.1 I 77/ 0022034515580796 jdr.sagepub.com

\author{
M. Khatibi Shahidi ${ }^{*}$, J. Krivanek ${ }^{2 *}$, N. Kaukua ${ }^{3}$, P. Ernfors ${ }^{4}$, L. Hladik ${ }^{5}$, \\ V. Kostal ${ }^{5}$, S. Masich ${ }^{6}$, A. Hampl $^{2}$, V. Chubanov ${ }^{7}$, T. Gudermann ${ }^{7}$, R.A. Romanov ${ }^{4}$, \\ T. Harkany ${ }^{4,8}$, I. Adameyko ${ }^{8,9}$, and K. Fried ${ }^{3}$
}

\begin{abstract}
In organized tissues, the precise geometry and the overall shape are critical for the specialized functions that the cells carry out. Odontoblasts are major matrix-producing cells of the tooth and have also been suggested to participate in sensory transmission. However, refined morphologic data on these important cells are limited, which hampers the analysis and understanding of their cellular functions. We took advantage of fluorescent color-coding genetic tracing to visualize and reconstruct in 3 dimensions single odontoblasts, pulp cells, and their assemblages. Our results show distinct structural features and compartments of odontoblasts at different stages of maturation, with regard to overall cellular shape, formation of the main process, orientation, and matrix deposition. We demonstrate previously unanticipated contacts between the processes of pulp cells and odontoblasts. All reported data are related to mouse incisor tooth. We also show that odontoblasts express TRPM5 and Piezo2 ion channels. Piezo2 is expressed ubiquitously, while TRPM5 is asymmetrically distributed with distinct localization to regions proximal to and within odontoblast processes.
\end{abstract}

Keywords: pulp biology, dentinogenesis, growth/development, protein expression, transgenic animals, cell morphology

\section{Introduction}

The tooth is built by highly organized and specialized cell types, including odontoblasts, ameloblasts, and cementoblasts. Production of dentin by odontoblasts is a key process that is crucial for tooth formation and regeneration. The odontoblasts form a single layer of postmitotic polarized cells at the periphery of the dental pulp, where they secrete the components of predentin-dentin. Their shielded localization with processes embedded in dentinal tubules has always complicated a detailed structural analysis of their morphology and organization. Furthermore, odontoblasts are not static but undergo significant morphologic changes during their life span. These can be divided into 4 stages reflecting their functionality: preodontoblast, secretory, transitional, and aging (Masthan 2010). Initial odontoblast cell differentiation occurs at the site of the epithelial-mesenchymal junction, which later becomes the dentin-enamel junction (DEJ). Some cytoplasmic vertical processes are emitted, which are lost as the odontoblast matures and are replaced by the main process that will become the secretory pole of the cell. However, the details of these transformations remain unknown (Yamada et al. 1983; Sasaki and Garant 1996; Arana-Chavez and Massa 2004). The length of the odontoblast process has been an issue of controversy. Some studies indicate that the process does not reach farther than halfway through the dentin (Thomas 1979), while others claim that the process reaches as far as the DEJ (Yamada et al. 1983). Side branches of odontoblasts have also been visualized at high microscopic resolution, and these are believed to be important for communication with the dentin matrix.

\footnotetext{
'Department of Dental Medicine, Karolinska Institutet, Huddinge, Sweden

${ }^{2}$ Department of Histology and Embryology, Faculty of Medicine, Masaryk University, Brno, Czech Republic

${ }^{3}$ Department of Neuroscience, Karolinska Institutet, Stockholm, Sweden ${ }^{4}$ Unit of Molecular Neurobiology, Department of Medical Biochemistry and Biophysics, Karolinska Institutet, Stockholm, Sweden

${ }^{5}$ TESCAN ORSAY Holding, Brno, Czech Republic

${ }^{6}$ Department of Cell and Molecular Biology, Karolinska Institutet, Stockholm, Sweden

${ }^{7}$ Ludwig-Maximilians-Universität München, Walther-Straub-Institut für Pharmakologie und Toxikologie, München, Germany

${ }^{8}$ Department of Molecular Neurosciences, Center of Brain Research, Medical University of Vienna, Vienna, Austria

'Department of Physiology and Pharmacology, Karolinska Institutet, Stockholm, Sweden

*Authors contributing equally to this article.
}

A supplemental appendix to this article is published electronically only at http://jdr.sagepub.com/supplemental.

\section{Corresponding Authors:}

K. Fried, Department of Neuroscience, Karolinska Institutet, Retzius vag 8, SE-I7I 77 Stockholm, Sweden.

Email: kaj.fried@ki.se

I. Adameyko, Department of Physiology and Pharmacology, Karolinska Institutet, SE-17I 77 Stockholm, Sweden.

Email: igor.adameyko@ki.se 
Several lines of evidence have pointed to the likelihood that the odontoblasts have a role in sensory transduction from teeth, although this remains to be clarified (Magloire et al. 2009). $\mathrm{Ca}^{2+}$ levels within the odontoblast increase upon mechanical stimuli, and odontoblasts possess mechano- and thermosensory ion channels as well as voltage-gated $\mathrm{Na}^{+}, \mathrm{Ca}^{2+}$, and $\mathrm{K}^{+}$ channels (reviewed by Chung et al. 2013). Recent data support the concept that odontoblasts could transmit external stimuli to pulpal nerve fibers equipped with purinergic receptors, through a release of ATP (Ichikawa et al. 2012; Shibukawa et al. 2015). Taken together, these results highlight the importance of knowledge on the formation and function of the process that traverses the dentin for the understanding of matrix deposition and putative sensory functions of odontoblasts.

Interestingly, it has been suggested that odontoblasts can act as a functional network if subjected to various stimuli. Odontoblasts have numerous specialized junctions, which allow for direct intercellular communication (Ruch 1998). Such junctions are also found between the odontoblasts and cells of the subodontoblast zone (see Goldberg and Smith 2004), suggesting that communication takes place between these compartments. The possibility that the subodontoblast zone is a source of progenitor cells for the odontoblast layer has not yet been fully addressed (see Goldberg and Smith 2004). A recent study on human teeth suggested that the subodontoblast zone and the odontoblasts constitute an intricate neurosensory structure in close proximity to the microvasculature, with properties similar to the blood-brain barrier (Farahani et al. 2011). It is relevant to ask whether specialized cellular domains and morphologic adaptations exist to combine odontoblasts and the subodontoblast pulp cell layer into an integrated network. Obviously, more work is necessary to clarify this issue as well as to elucidate how odontoblasts mature, how they deposit matrix, and how they respond to external stimuli. Structural studies represent vital elements of such investigations.

Recent advances in microscopy, image analysis software, and transgenic animal models have provided us with state-ofthe-art tools to address issues regarding tooth growth with high-resolution imaging in 3-dimensional (3D) settings. Using such techniques, we have shown that Schwann cell precursors and Schwann cells are recruited from nerves and contribute to the formation of parasympathetic ganglia but also, interestingly, give rise to dental pulp cells and odontoblasts (Dyachuk et al. 2014; Kaukua et al. 2014). Here, we have taken advantage of this type of genetic tracing to label individual mouse incisor odontoblasts. Compilations of 3D images of odontoblasts have yielded novel data on cell shape, orientation, pruning of processes, and other aspects of spatial organization in conjunction with the subodontoblastic cell layer. We also provide insights into possible mechanisms of progressive matrix deposition during tooth growth by showing the dynamic changes of odontoblast processes during their maturation. On the basis of our data, we suggest a new model that describes how the main process of a mature mouse incisor odontoblast is selected out of a wealth of thin processes, many of which are initially in contact with ameloblasts.

\section{Materials and Methods}

\section{Animals}

All animal work was approved by the Ethical Committee on Animal Experiments (Stockholm North Committee) and performed according to the Swedish Animal Agency's provisions and guidelines for animal experimentation. SOX10-CreERT/ R26YFP (Laranjeira et al. 2011) and PLP-CreERT2/R26YFP mice were used for genetic cell lineage tracing of peripheral glia (Leone et al. 2003). R26Confetti mice were received from the laboratory of H. Clevers, Hubrecht Institute, Utrecht, The Netherlands, and bred with the PLP-CreERT2 strain to obtain PLP-CreERT2/R26Confetti offspring for clonal analysis. Genetic recombination was induced by tamoxifen (Sigma T5648) dissolved in corn oil (Sigma C8267), administered through intraperitoneal injection at a maximum dosage of $5 \mathrm{mg} / \mathrm{kg}$ (at times given repeatedly over periods of days/weeks to gain more robust recombination). Tracing time varied between $42 \mathrm{~d}$ and 1 y postinjection.

\section{Tissue Handling and Antibodies}

The animals were sacrificed by an overdose of isoflurane and perfused with phosphate buffered saline, followed by $4 \%$ paraformaldehyde. Maxillary and mandibular adult incisors were carefully dissected out, cryopreserved, and embedded in OCT medium. For descriptions of subsequent immunohistochemistry and imaging, see Materials and Methods in the Appendix.

\section{Sample Preparation for Focused Ion Beam- Scanning Electron Microscopy Analysis}

All focused ion beam (FIB)-scanning electron microscopy (SEM) investigations were performed with FERA3 plasma FIBSEM in collaboration with TESCAN, Brno, Czech Republic. For details, see Materials and Methods in the Appendix.

\section{Results}

\section{Morphologic Features of the Odontoblast Layer}

To address the 3D morphology of odontoblasts, we employed genetic tracing with multicolored reporters that label cells with combinations of fluorescent proteins (Snippert et al. 2010). Specifically we used the PLP-CreERT2/R26Confetti mouse strain and the associated methodology. We visualized the clonal properties of glial cells as they contributed to tissue and organ formation. After several weeks of genetic tracing, individually labeled dental mesenchymal stem cells give rise to different cohorts of odontoblasts that occupy adjacent territories or intermingle at borders (Fig. 1A-C). We used sections from genetically traced nondecalcified adult teeth to obtain high-quality images and 3D reconstructions of separate colorcoded odontoblasts and their assemblages. In their terminal parts, we found that the cylindrical cell bodies were constricted and formed bottleneck-like structures. The cell bodies then 


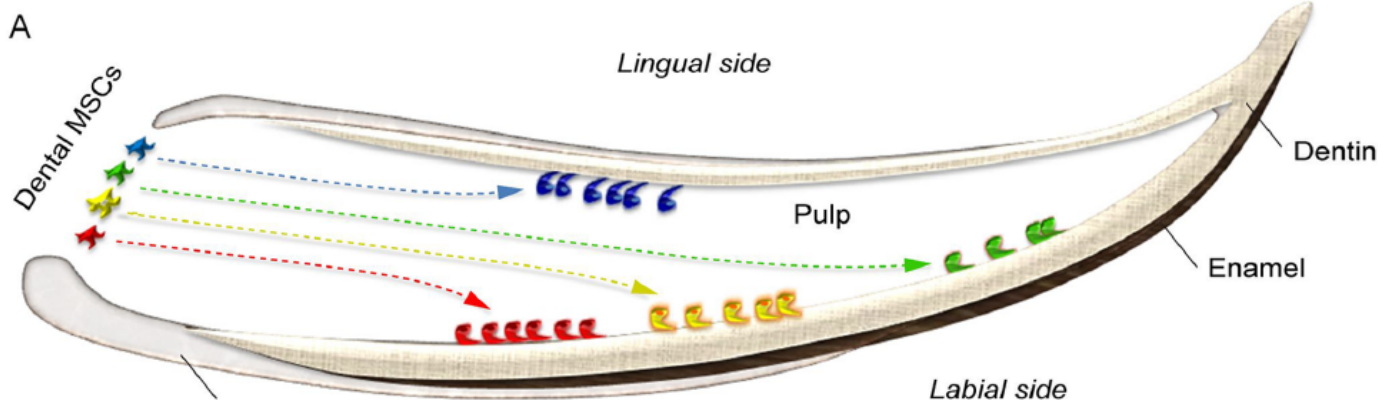

Enamel organ

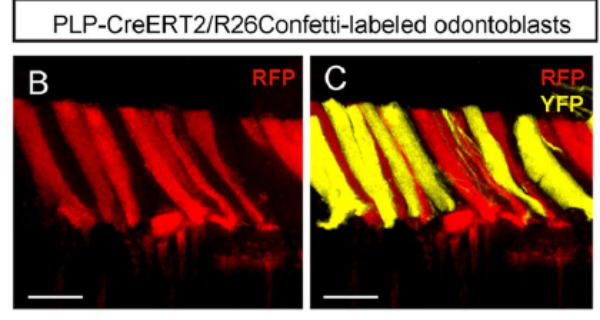

Odontopodes and odontoblast processes
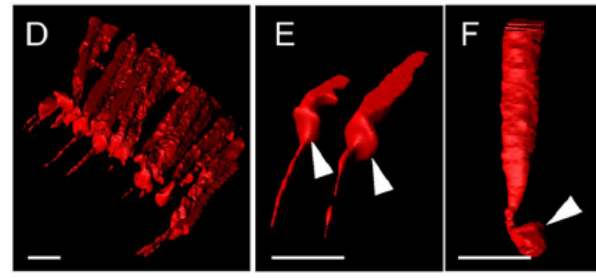

L

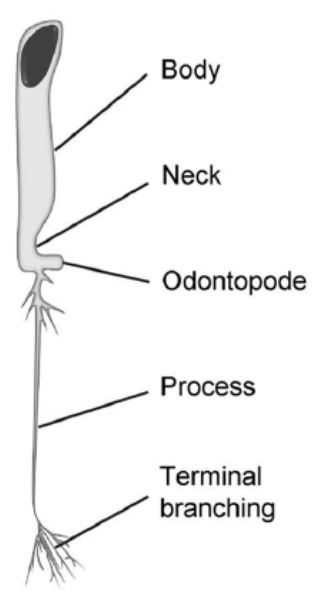

M
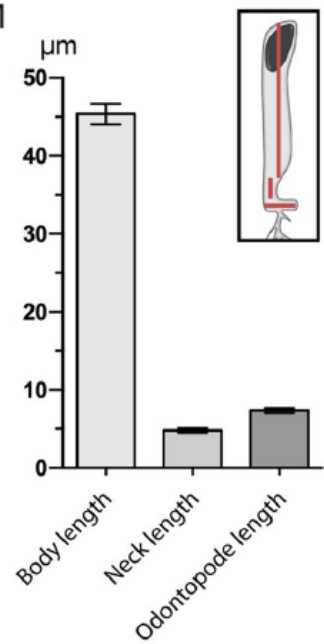

3D-rendered isosurfaces around odontoblasts
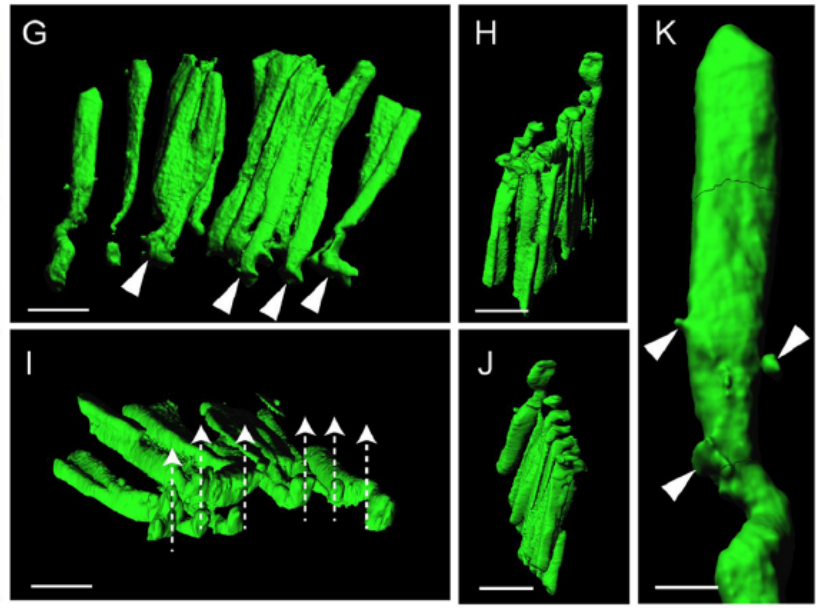

N
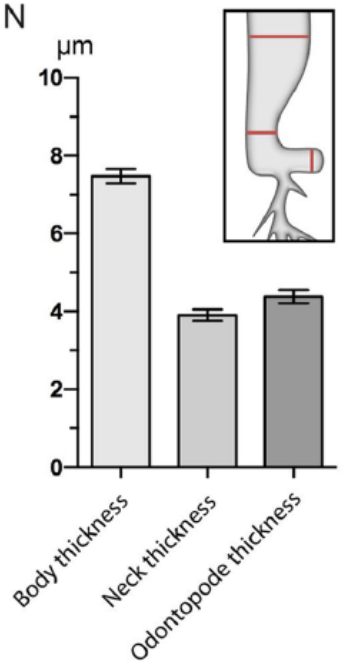

Figure I. Three-dimensional (3D) rendering of clonally color-coded odontoblasts in genetically traced PLP-CreERT2/R26Confetti animals. (A) Schematic illustration showing dental mesenchymal stem cells (MSCs; color coded) giving rise to clusters of localized odontoblasts. (B, C) YFP ${ }^{+}$- and RFP $^{+}$-traced odontoblasts from 40-d traced PLP-CreERT26/R26Confetti mouse incisor. (D-F) Three-dimensional isosurfaces reveal the fine structure of genetically traced odontoblasts with distinct narrowing of the body into a bottleneck-like constriction that further expands into the odontopode compartment adjacent to the dentin (pointed out by arrowheads in $E$ and F). (G-K) Three-dimensional rendering of odontoblasts shows how these cells are organized into cohorts with uniformly oriented odontopodes (arrowheads). Arrows point out the direction of odontopode orientation toward the incisal part of the tooth. (K) Magnification of rendered odontoblast surface with visible irregular structures (arrowheads). (L) Schematic illustration shows cellular compartments and asymmetric arrangements of odontoblasts. Structure of adult terminal branches is predicted from dentinal tubule patterns. (M) Graph demonstrating the variation of lengths of different cellular compartments (outlined in red in inset; scanning electron microscopy: body, $45.36 \pm$ I. $3 \mu \mathrm{m}$; neck, $4.78 \pm 0.3 \mu \mathrm{m}$; odontopode, $7.342 \pm 0.3 \mu \mathrm{m} ; n=20)$. ( $\mathbf{N}$ ) Graph showing the diameters of different cellular compartments (outlined in red in inset; scanning electron microscopy: body, $7.47 \pm 0.2 \mu \mathrm{m} ;$ neck, $3.9 \pm 0.1 \mu \mathrm{m}$; odontopode, $4.38 \pm 0.2 \mu \mathrm{m} ; n=20$ ). Scale bars: I $5 \mu \mathrm{m}(\mathrm{B}-\mathrm{J}, \mathrm{L}), 5 \mu \mathrm{m}(\mathrm{K})$. 
again expanded into previously undefined compartments from which main processes emanated (Fig. 1D, F). We termed these footlike compartment structures odontopodes, which appeared to be stereotypically and asymmetrically angulated structures that face the tip of the incisor and partially overlap in scalelike pattern (Fig. 1G-N). This rendered the odontoblasts polarized and oriented along the anterioposterior axis of the tooth. Such orientation was additionally highlighted by the specific angulations of mature odontoblasts and an angle of the dentinal tubules as they enter the matrix (Fig. 1E). We found that the main process of the odontoblast is always emitted from the most anterior terminal part of the odontopode (Fig. 1L-N). Some additional minor filaments branching out of the main process were also detected. Odontopodes, although always present in mature cells, demonstrated some degree of variation in their qualitative morphology.

In the mature odontoblast layer, we also detected genetically traced cells with a drastically different shape: these were pyramid-shaped cells with a broad base in contact with the predentin (Fig. 2A-D, H). The nuclei of these cells were located in the region adjacent to the dentin matrix, as opposed to the basally positioned nuclei of the odontoblasts. We could not determine whether these cells had any process that entered the matrix. Our genetic tracing demonstrated that they are derived from dental mesenchymal stem cells, but their functional role and relation to other known cell types in the tooth remain elusive. Next, we used immunocytochemistry with antibodies against NG2, a marker for pericytes (Feng et al. 2011), and the FIB-SEM technique to demonstrate that blood vessels typically traversed the odontoblast layer between cellular constrictions proximal to the predentin (Fig. 2A-H, Supplemental Movie 1).

\section{Dynamics of Odontoblast Maturation and Generation of the Main Process}

Odontoblast maturation manifests in the generation of 1 main process that will traverse between the matrix and the DEJ. However, morphologic details of this process are largely unknown. We found that pre- and immature odontoblasts demonstrate asymmetric morphology in the anterioposterior direction, starting when the first supernumerary thin processes develop at the terminal surface of the cell. These processes formed a dense brush and were emitted from the apical portion of the odontoblast that faces the coronal end of the tooth. The diameter of the processes was $346.5 \pm 13.0 \mathrm{~nm}$ (SEM; $n=30$ ), and the length of the processes was $6.0 \pm 0.4 \mu \mathrm{m}$ (SEM; $n=30$; Fig. $3 \mathrm{~A}-\mathrm{E}$ ). The processes did not seem to be specifically oriented or arranged. They likely serve to massively increase the apical surface of the odontoblast, thus facilitating an efficient and rapid deposition of matrix. We did not observe branching of these thin processes or major differences in length and thickness. Importantly, many of the thin processes were in very close proximity to ameloblasts (Fig. 3A-C) and so may be involved in signal transmission that coordinates maturation of odontoblasts and ameloblasts (Dassule et al. 2000). Additionally, at this stage, the main body of the odontoblast began to assume an angulated position in relation to the plane of matrix deposition (Fig. 3J). Next, mineral accumulated around the submerged terminal section adjacent to the DEJ, while cellular growth and elongation continued inward toward the pulp core from the basal region (Fig. 3F-J). Gradually, the odontopode took shape as the main odontoblast processes and its side branches, which were absent at previous phases, acquired more distinct morphologies (Fig. 3K-Q). After the first layers of matrix were deposited, the entire brushlike terminal part of the odontoblasts appeared to become submerged into the matrix (Fig. 3M-Q, Supplemental Movies 2 and 3). From this stage onward, the odontoblasts could be considered as morphologically mature, since the main processes were surrounded by newly formed dentinal tubules with multiple side channels (Fig. 3N, O).

\section{Piezo2 and TRPM5: 2 Novel Odontoblast Proteins}

Many membrane proteins in most cell types are restricted to specialized compartments of the membrane (Aimon et al. 2014). However, with the exception of junction proteins, such data have so far not been reported with regard to odontoblasts. Our results demonstrated that TRPM5 not only is asymmetrically distributed in odontoblasts already at an immature stage (Fig. 4A) but also remains expressed in mature cells with distinct localization to odontopodes (Fig. 4A, B) and odontoblast processes (Fig. 4C). Next, we found that the evolutionarily conserved mechanosensory ion channel Piezo2 (Coste et al. 2012) was also expressed in odontoblasts (Fig. 4D-F). However, the pattern of Piezo2 distribution was distinctly different from that of TRPM5, being rather ubiquitous. Using TRPM5 labeling, we detected sparse membrane protrusions, some of which appeared to extend inwardly toward the pulp (Fig. 4E, F; white arrows in Fig. 5E).

\section{Numerous but Not All Processes from the Pulp Cells Penetrate into the Odontoblast Layer}

Morphologic signs that odontoblasts and pulp cells in subodontoblast layers are functionally integrated have not been determined at the level of multicellular assemblages. The genetic tracing-based color-coding of odontoblasts and pulp cells allowed us to label and analyze clusters of odontoblasts in 1 color, with adjacent pulp cells marked by a different color (Fig. 5A-D). We found that pulp cells from the subodontoblast layer emit some processes deep into the odontoblast layer and directly contact the cell membranes of odontoblasts body and odontopodes (Fig. 5E-H). Multiple long processes from a single pulp cell in the subodontoblast layer contacted several separate odontoblasts (Fig. 5A, E, H). As mentioned above, some of our observations also suggested that odontoblasts emitted thin basal processes directed toward the subodontoblast region (Fig. 5E-G). Overall, odontoblasts displayed multiple surface irregularities that potentially facilitate odontoblastsubodontoblast cell interactions. Pulp cells in general presented multiple branches going in all directions (mean, 4.4 main branches - which in turn emitted further subbranches; 

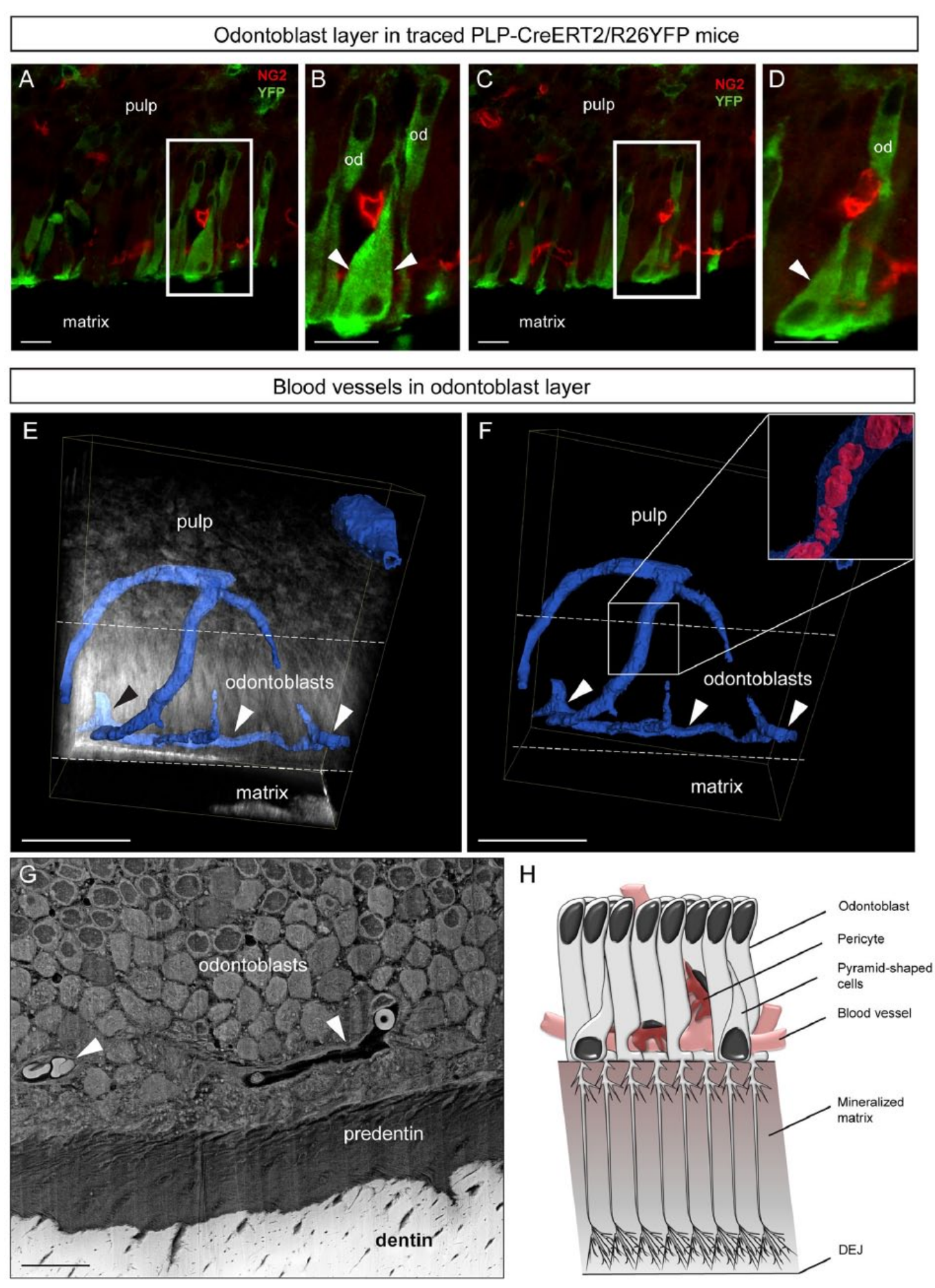

Figure 2. Unusual morphologically distinct cells and blood vessels identified in the odontoblast layer near dentin matrix. Panels $\mathbf{A}$ and $\mathbf{C}$ show the layer of mature odontoblasts (od) from 30-d traced PLP-CreERT2/R26YFP mouse incisor, and panels B and D are magnified regions, respectively. NG2 as pericyte marker displays position of vessels inside the genetically traced odontoblast layer. Arrowheads point at unusual morphologically distinct pyramid-shaped $\mathrm{YFP}^{+}$cells in the odontoblast layer. Panels $\mathbf{E}$ and $\mathbf{F}$ are 3-dimensional visualizations of blood vessels near and inside the odontoblast layer. Arrowheads point out blood vessels adjacent to the matrix in the odontoblast neck region. Framed image in panel $\mathrm{F}$ shows erythrocytes (red) inside a blood vessel (blue). Visualization was obtained from focused ion beam-scanning electron microscopy stack and compiled using ORS Visual software. (G) Scanning electron microscopy image from tilted section of odontoblast layer (neck region) with blood vessels. $(\mathbf{H})$ Schematic drawing of the complex organization of the incisor odontoblast layer. Structure of terminal branches is predicted from dentinal tubule patterns. Scale bars: $15 \mu \mathrm{m}$ $(A-D, G), 15 \mu \mathrm{m}(E, F)$. DEJ, dentin-enamel junction. 

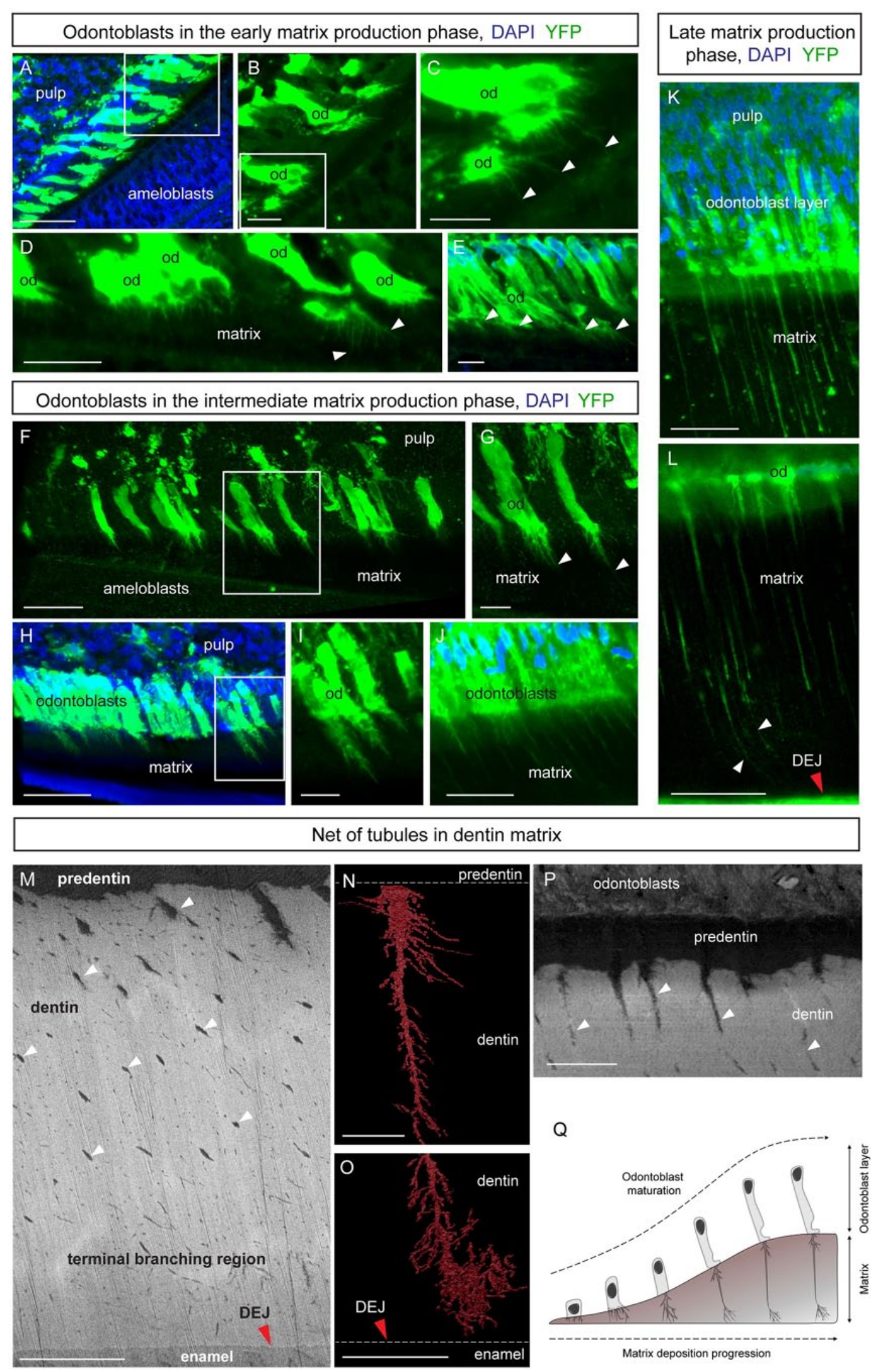

Figure 3. Morphologic changes during maturation of the odontoblasts. (A-E) YFP ${ }^{+}$genetically traced immature odontoblasts from PLP-CreERT2/ R26YFP mouse incisor. Note that the genetic labeling does not label all odontoblasts in the odontoblast layer. Multiple thin processes emanate from brushlike compartment and course inside the matrix toward ameloblasts. Panel B shows the magnified area outlined by rectangle in panel A. Panel C demonstrates the magnified area outlined in panel B. Arrowheads in $C$ point out at the processes that reach the ameloblasts layer. Arrowheads in $D$ and $\mathrm{E}$ show the matrix-adjacent compartment equipped with asymmetrically oriented brushlike structures. Note the angulated position of immature odontoblasts in respect to the plane of deposited matrix. (F-J) YFP ${ }^{+}$genetically traced odontoblasts in intermediate matrix production phase. Panel $\mathrm{G}$ shows the magnified area outlined in panel F. Arrowheads point out at the prominent odontoblast process with numerous side branches. Panel I represents the magnified area from panel $\mathrm{H}$. Panel J shows further transformation of odontoblast processes, and IMARIS clipping plane is introduced to show free projection of odontoblast process entry into the dentinal tubule; same region is visualized as a merged stack in panel L. (K, L) YFP ${ }^{+}$ genetically traced odontoblasts in their late matrix production phase with a single long process emitted into the matrix. Arrowheads point out a 
Fig. 5A, I). Pulp cell processes that were opposed to odontoblasts appeared to be morphologically distinct, since they were on average longer; furthermore, branching was largely discontinued after contacting the odontoblasts (Fig. 5I). Taken together, these data support a model where pulpal subodontoblast cells and the odontoblast layer are highly integrated. Further studies are required to address the functionality of this cellular network.

\section{Discussion}

In this study, we demonstrate novel features of the 3D structure of mouse incisor odontoblasts, using a combination of advanced lineage tracing and confocal microscopy techniques. We also demonstrate for the first time (to the best of our knowledge) that odontoblasts express TRPM5 and Piezo2 ion channels, which could underpin dental mechanosensation. These findings may have important implications for the functional understanding of local odontoblast networks and interactions, as well as odontoblast-nerve communication.

We show that the odontoblast has a slender oblong cell body, which in its terminal region tapers off into a bottleneck-like constriction. The bottleneck bends in an almost 90-degree angle, widens again, and forms a footlike structure that we termed odontopode, from which the main process protrudes. We have introduced the novel designation odontopode since we saw a need for a proper description of this distinct anatomic region of the odontoblast. With regard to its specific function, further studies may show in what way it might influence dentin formation or other aspects of odontoblast activities. In principle, the odontopode might be subject to different interpretations, and time will tell if this nomenclature refers appropriately to a structure that will be independently verified by other investigators using complementary approaches.

Our data suggest that, at least in the mouse incisor, the bottleneck constriction between the odontopode and the main body of the odontoblast represents appropriate adaptation to provide space for a blood vessel network. A specifically organized system for oxygen and metabolite supply should be of ultimate importance for the long processes of odontoblasts, since, unlike bone, dentin and enamel are not vascularized (Goldberg 2014). We also found protrusions directed toward the pulp, which could function as, for example, morphogen gradient-sensing elements (Kornberg 2014) in interactions with the subodontoblastic cell layer or as transport routes for nutrients from the pulp enriched in microvessels.

We also demonstrate that the odontoblast does not develop merely 1 process during tooth growth (Tominaga et al. 1984; Couve 1986; Sasaki and Garant 1996; Goldberg 2014). Instead, it initially emits multiple brushlike thin extensions, and it is only later that just 1 main process remains (Mjor and Nordahl 1996). However, using FIB-SEM, we show that after maturation, interlaced networks composed of multiple thin tubules can be still found near the DEJ, thus reflecting the historical brushlike arrangement of multiple odontoblast processes.

location where the processes change their trajectory in the matrix. (M-P) Scanning electron microscopy and detailed visualization of fully mineralized dentin matrix with network of dentinal tubules. Panel $M$ shows dentin matrix sectioned by focused ion beam (FIB) with main tubule and the terminal branching region. Panels $\mathrm{N}$ and $\mathrm{O}$ represent compilations of a single dentinal tubule in near-predentin (N) and near-DEJ (O) regions, respectively, from data obtained by FIB-scanning electron microscopy. Panel P shows initial parts of wide and branched processes in the initial layer of dentin matrix obtained from FIB-scanning electron microscopy stack using clipping plane in ORS Visual software. White arrowheads in panels $M$ and $P$ indicate the main thick parts of dentinal processes within the dentin matrix. (Q) Schematic illustration of morphologic changes during maturation of odontoblasts. Structure of adult terminal branches is predicted from dentinal tubule patterns. Scale bars: $50 \mu \mathrm{m}(\mathrm{A}, \mathrm{F}, \mathrm{H}, \mathrm{J}-\mathrm{L})$, I5 $\mu \mathrm{m}$ (B-E, G, I, M-P). DEJ, dentinenamel junction; od, odontoblast. 

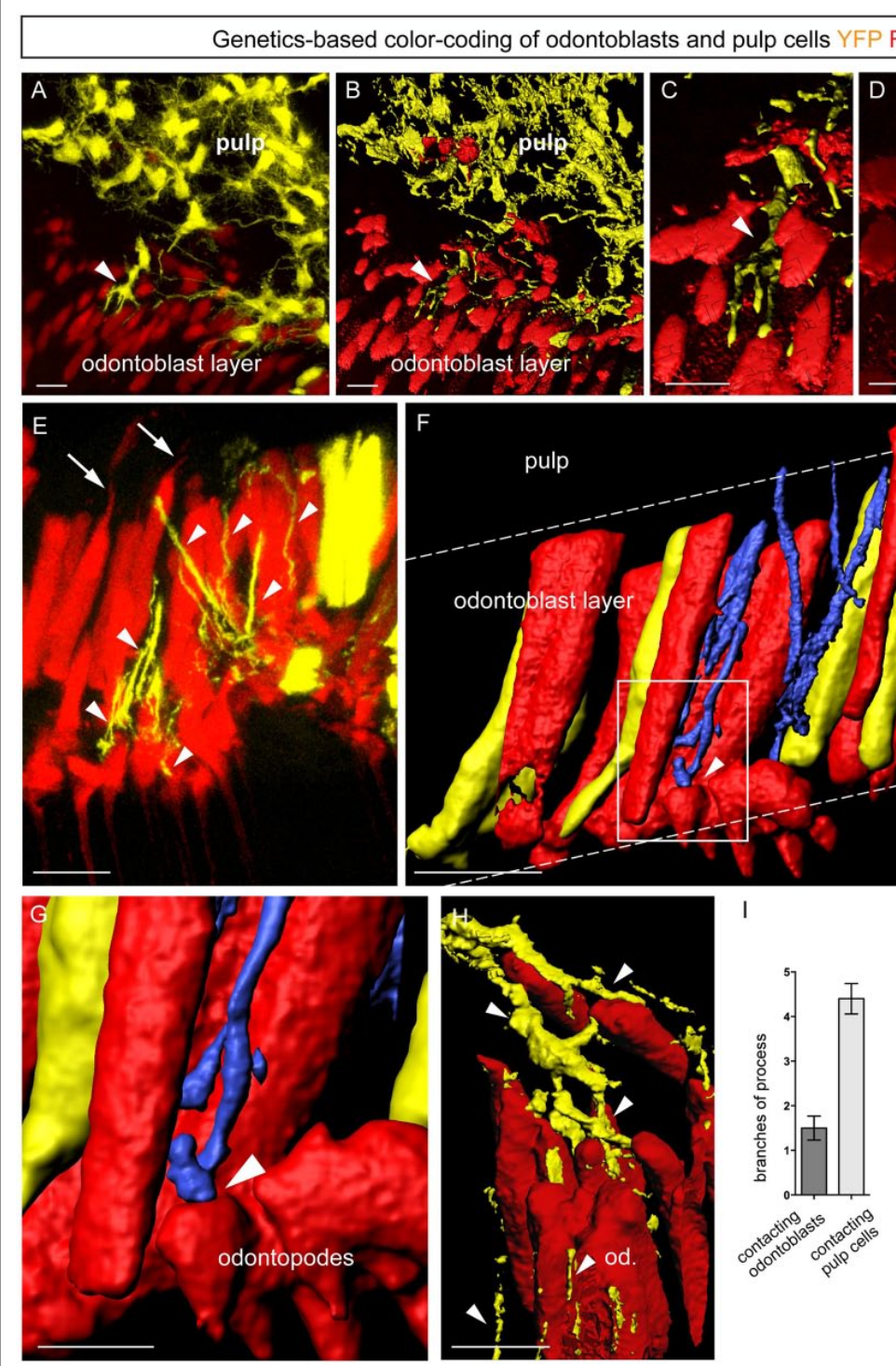

Figure 5. Pulp cells in the subodontoblast region contact odontoblasts via distinct processes. Projection of confocal stack showing $\mathrm{YFP}^{+}$and $\mathrm{RFP}^{+}$genetically traced pulp cells and odontoblasts. (A) The pulp cells show extensive branching of their multiple processes, highlighted by the cell marked by an arrowhead. (B-D) Arrowheads point at the cell marked in panel A but visualized from different angles. Note 5 distinct YFP $^{+}$processes within the odontoblast layer. (E, H) Arrowheads indicate the $\mathrm{YFP}^{+}$processes of pulp cells entering the odontoblast layer. Arrow in panel $\mathrm{E}$ points out the protrusions from odontoblasts that are oriented toward the pulp. Panels $\mathbf{F}$ and $\mathbf{G}$ are rendered 3-dimensional isosurfaces from panel E, with the pulpal cells processes shown in blue. Arrowhead in panel $\mathrm{G}$ marks a close apposition between pulp cell process and an odontopode. (I) Graph showing the difference in length and branching pattern of those processes that enter the odontoblast layer and those that do not. Left graph, scanning electron microscopy: processes entering odontoblast layer give rise to 1.5 branches $\pm 0.3(n=10)$; nonentering processes give rise to 4.4 branches $\pm 0.3(n=10)$. Right graph, scanning electron microscopy: length of the process entering odontoblast layer is $36.65 \pm 1.7 \mu \mathrm{m}$ $(n=10)$, while the length of the process that does not enter odontoblast layer is $22.9 \pm 1.6 \mu \mathrm{m}$ $(n=44)$. Scale bars: $15 \mu \mathrm{m}(\mathrm{A}-\mathrm{F}, \mathrm{H}), 5 \mu \mathrm{m}(\mathrm{G})$.

These tubules in fully formed dentin contain very thin odontoblast processes and probably represent remnants of the early dentinogenesis. Thus, some primitive brushlike extensions have likely been trapped within the matrix at the initial deposition of hard matrix. The functional role, if any, of these networks
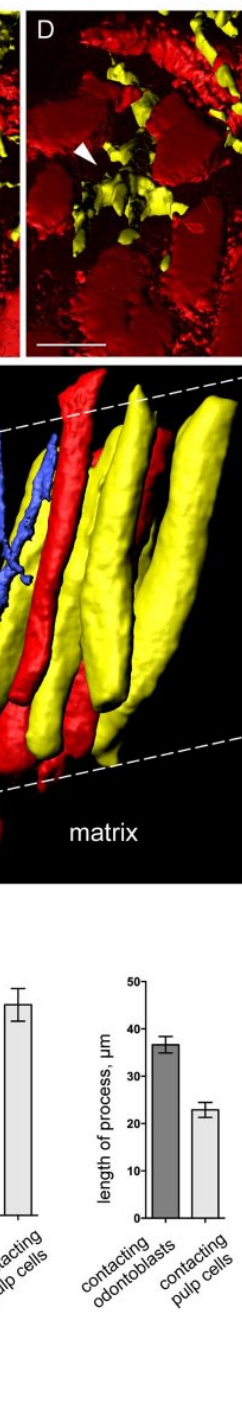

remains unclear. Hypothetically, they could be involved in sensory mechanisms and influence dentin sensitivity. Our data strongly suggest that the main process forms as a result of stretching and lengthening of the large terminal asymmetric compartment of pre- and immature odontoblasts and not through pruning and selection of single processes out of the many that are initially generated. On this basis, we hypothesize that after differentiation of the terminal compartment into the odontopode and the main process, the latter performs exclusively sensory functions while further deposition of matrix is performed by the odontopode. This hypothesis, of course, needs further investigation.

We have identified pyramidshaped cells in the odontoblast layer with their nuclei situated in their broad base near the predentin. This is in contrast to the odontoblasts, which have their nuclei in their basal region. Since they were traced, they were of the same lineage as odontoblast (i.e., Schwann cell precursor derived). Being located near capillaries and odontopodes, they might have a supportive role for odontoblasts and especially for the highly active odontopode region. However, their role remains elusive.

In addition to the major function of odontoblasts in dentin production, several studies suggested that the odontoblasts have properties of sensory cells (Chung et al. 2013). In response to TRP channel (TRPV1, TRPV2, TRPV4, and TRPA1) activation, odontoblasts can release ATP through the ATP-permeable channel pannexin 1. Adjacent pulpal nerve endings could receive this signal through P2X receptors. Expression of pannexin 1 is uniform in the odontoblast body but predominantly in terminal parts (Shibukawa et al. 2015). TRPA1, which is thermosensitive, is expressed in terminal parts of odontoblasts and in processes (Tsumura et al. 2013). Another TRP family member, TRPM 5 , is a $\mathrm{Ca}^{2+}$ activated calcium impermeable channel, which transforms initial calcium response into depolarization (Liu and Liman 2003; Prawitt et al. 2003). TRPM5 plays a prominent role in taste 
reception, where it is a part of a signal transduction system (Liu and Liman 2003). $\mathrm{Ca}^{2+}$-dependent activation of TRPM5 results in a depolarizing generator potential and subsequent ATP release through voltage-gated ATP-permeable channels (Romanov et al. 2007), formed by the recently discovered transmembrane protein CALHM1 (Taruno et al. 2013). We found that TRPM5 was expressed in the main odontoblast process and the odontopode. This suggests that those cellular regions may represent specialized sensory compartments (Aimon et al. 2014) . Here, depolarization could occur due to the opening of TRPM5 in response to local calcium increasesfor example, after activation of calcium-permeable thermoand mechanosensitive TRP channels in odontoblasts. We also revealed that Piezo2 ion channel is expressed in all regions of the odontoblast. Piezo2 forms a pore in a membrane and produces cation currents in response to mechanical stimulation. Piezo channels play an important role in mechanosensation in Merkel cells (Coste et al. 2012; Ikeda et al. 2014; Woo et al. 2014) and in shear stress in blood vessels, urinary bladder, and urethra, where mechanical stimulation is important for development and function (Bagriantsev et al. 2014). The function of Piezo2 in odontoblasts, however, remains to be demonstrated.

Importantly, the reported results are obtained exclusively for mouse incisor, which is one of the best model systems to study molecular mechanisms of different processes. However, the mouse incisor is a continuously growing tooth (about $450 \mu \mathrm{m} / \mathrm{d}$ ) where odontoblasts are highly packed within the layer and terminal blood vessels are located within the odontoblastic layer. Such specializations may account for possible structural dissimilarities with odontoblasts from mouse and human molars or other teeth that are not continuously growing.

In conclusion, we present a detailed microarchitecture of odontoblasts and associated cells during development and in fully formed tissue. Based on the odontoblast expression of the ion channels TRPM5 and Piezo2, our findings reinforce the hypothesis that odontoblasts might have a sensory function. Specifically, this could highlight their capacity as mechanosensors of the tooth pulp.

\section{Author Contributions}

M. Khatibi Shahidi, contributed to conception and design, drafted the manuscript; J. Krivanek, N. Kaukua, contributed to conception, design, and data analysis, drafted the manuscript; P. Ernfors, L. Hladik, V. Kostal, S. Masich, A. Hampl, V. Chubanov, T. Harkany, contributed to data analysis, critically revised the manuscript; T. Gudermann, R.A. Romanov, contributed to data analysis, drafted the manuscript; I. Adameyko, K. Fried, contributed to conception, design, and data analysis, drafted and critically revised the manuscript. All authors gave final approval and agree to be accountable for all aspects of the work.

\section{Acknowledgments}

This study was supported by the Swedish Research Council (I.A., K.F., P.E.), the Bertil Hallsten Research Foundation (I.A.), StratRegen and the Wallenberg Foundation (CLICK [Center for Live Imaging of Cells at Karolinska Institutet]; I.A.), a Wallenberg Scholar and European Research Council advanced grant (P.E.), the National Graduate School in Odontological Science (M.K.S.), the Swedish Dental Association (M.K.S., N.K), Czech Ministry of Education Youth and Sports (J.K.), European Social Fund in the Czech Republic (A.H.), Cooperation between Masaryk University and Karolinska Institutet Stockholm in the field of biomedicine (CZ.1.07/ 2.3.00/20.0180; J.K., A.H.), the Faculty of Medicine of Masaryk University (MUNI/A/1014/2013; J.K., A.H.), the Stockholm County Council (N.K.), and Novo Nordisk Foundation (T.H.). Special thanks to the TESCAN ORSAY holding, Brno, Czech Republic (L.H., V.K.). The authors declare no potential conflicts of interest with respect to the authorship and/or publication of this article.

\section{References}

Aimon S, Callan-Jones A, Berthaud A, Pinot M, Toombes GE, Bassereau P. 2014. Membrane shape modulates transmembrane protein distribution. Dev Cell. 28(2):212-218.

Arana-Chavez VE, Massa LF. 2004. Odontoblasts: the cells forming and maintaining dentine. Int J Biochem Cell Biol. 36(8):1367-1373.

Bagriantsev SN, Gracheva EO, Gallagher PG. 2014. Piezo proteins: regulators of mechanosensation and other cellular processes. J Biol Chem. 289(46):31673-31681.

Chung G, Jung SJ, Oh SB. 2013. Cellular and molecular mechanisms of dental nociception. J Dent Res. 92(11):948-955.

Coste B, Xiao B, Santos JS, Syeda R, Grandl J, Spencer KS, Kim SE, Schmidt M, Mathur J, Dubin AE, et al. 2012. Piezo proteins are pore-forming subunits of mechanically activated channels. Nature. 483(7388):176-181.

Couve E. 1986. Ultrastructural changes during the life cycle of human odontoblasts. Arch Oral Biol. 31(10):643-651.

Dassule HR, Lewis P, Bei M, Maas R, McMahon A. 2000. Sonic hedgehog regulates growth and morphogenesis of the tooth. Development. 127:47754785 .

Dyachuk V, Furlan A, Shahidi MK, Giovenco M, Kaukua N, Konstantinidou C, Pachnis V, Memic F, Marklund U, Müller T, et al. 2014. Neurodevelopment: parasympathetic neurons originate from nerve-associated peripheral glial progenitors. Science. 345(6192):82-87.

Farahani RM, Simonian M, Hunter N. 2011. Blueprint of an ancestral neurosensory organ revealed in glial networks in human dental pulp. J Comp Neurol. 519(16):3306-3326.

Feng J, Mantesso A, De Bari C, Nishiyama A, Sharpe PT. 2011. Dual origin of mesenchymal stem cells contributing to organ growth and repair. Proc Natl Acad Sci U S A. 108(16):6503-6508.

Goldberg M. 2014. Pulp Vascularization and its regulation by the microenvironment. In: The dental pulp biology, pathology, and regenerative therapies. Berlin (Germany): Springer-Verlag. p. 61-71.

Goldberg M, Smith AJ. 2004. Cells and extracellular matrices of dentin and pulp: a biological basis for repair and tissue engineering. Crit Rev Oral Biol Med. 15(1):13-27.

Ichikawa H, Kim HJ, Shuprisha A, Shikano T, Tsumura M, Shibukawa Y, Tazaki M. 2012. Voltage-dependent sodium channels and calciumactivated potassium channels in human odontoblasts in vitro. $\mathrm{J}$ Endod. 38(10):1355-1362.

Ikeda R, Cha M, Ling J, Jia Z, Coyle D, Gu JG. 2014. Merkel cells transduce and encode tactile stimuli to drive Abeta-afferent impulses. Cell. 157(3):664-675.

Kaukua N, Shahidi MK, Konstantinidou C, Dyachuk V, Kaucka M, Furlan A, An Z, Wang L, Hultman I, Ahrlund-Richter L, et al. 2014. Glial origin of mesenchymal stem cells in a tooth model system. Nature. 513(7519):551-554.

Kornberg TB. 2014. The contrasting roles of primary cilia and cytonemes in $\mathrm{Hh}$ signaling. Dev Biol. 394(1):1-5.

Laranjeira C, Sandgren K, Kessaris N, Richardson W, Potocnik A, Vanden Berghe P, Pachnis V. 2011. Glial cells in the mouse enteric nervous system can undergo neurogenesis in response to injury. J Clin Invest. 121(9):3412-3424.

Leone DP, Genoud S, Atanasoski S, Grausenburger R, Berger P, Metzger D, Macklin WB, Chambon P, Suter U. 2003. Tamoxifen-inducible glia-specific Cre mice for somatic mutagenesis in oligodendrocytes and Schwann cells. Mol Cell Neurosci. 22(4):430-440.

Liu D, Liman ER. 2003. Intracellular Ca2+ and the phospholipid PIP2 regulate the taste transduction ion channel TRPM5. Proc Natl Acad Sci U S A. 100(25):15160-15165.

Magloire H, Couble ML, Thivichon-Prince B, Maurin JC, Bleicher F. 2009 Odontoblast: a mechano-sensory cell. J Exp Zool Part B. 312(5):416-424. 
Masthan KM. 2010. Textbook of human oral embryology, anatomy, physiology, histology and tooth morphology. New Delhi (India): JayPee Brothers Medical Publishing Ltd.

Mjor IA, Nordahl I. 1996. The density and branching of dentinal tubules in human teeth. Arch Oral Biol. 41(5):401-412.

Prawitt D, Monteilh-Zoller MK, Brixel L, Spangenberg C, Zabel B, Fleig A, Penner R. 2003. TRPM5 is a transient Ca2+-activated cation channel responding to rapid changes in $[\mathrm{Ca} 2+]$ i. Proc Natl Acad Sci U S A 100(25):15166-15171.

Romanov RA, Rogachevskaja OA, Bystrova MF, Jiang P, Margolskee RF, Kolesnikov SS. 2007. Afferent neurotransmission mediated by hemichannels in mammalian taste cells. EMBO J. 26(3):657-667.

Ruch JV. 1998. Odontoblast commitment and differentiation. Biochem Cell Biol. 76(6):923-938.

Sasaki T, Garant PR. 1996. Structure and organization of odontoblasts. Anat Rec. 245(2):235-249.

Shibukawa Y, Sato M, Kimura M, Sobhan U, Shimada M, Nishiyama A, Kawaguchi A, Soya M, Kuroda H, Katakura A, et al. 2015. Odontoblast as sensory receptors: transient receptor potential channels, pannexin-1, and ionotropic ATP receptors mediate intercellular odontoblast-neuron signal transduction. Pflugers Archiv. 467(4):843-863.
Snippert HJ, van der Flier LG, Sato T, van Es JH, van den Born M, KroonVeenboer C, Barker N, Klein AM, van Rheenen J, Simons BD, et al. 2010. Intestinal crypt homeostasis results from neutral competition between symmetrically dividing Lgr5 stem cells. Cell. 143(1):134-144.

Taruno A, Vingtdeux V, Ohmoto M, Ma Z, Dvoryanchikov G, Li A, Adrien L, Zhao H, Leung S, Abernethy M, et al. 2013. CALHM1 ion channel mediates purinergic neurotransmission of sweet, bitter and umami tastes. Nature. 495(7440):223-226

Thomas HF. 1979. The extent of the odontoblast process in human dentin. J Dent Res. 58(Spec Issue D):2207-2218.

Tominaga H, Sasaki T, Higashi S. 1984. Ultrastructural changes in odontoblasts during early development. Bull Tokyo Dent Coll. 25(1):9-26.

Tsumura M, Sobhan U, Sato M, Shimada M, Nishiyama A, Kawaguchi A, Soya M, Kuroda H, Tazaki M, Shibukawa Y. 2013. Functional expression of TRPM8 and TRPA1 channels in rat odontoblasts. PloS One. 8(12):e82233.

Woo SH, Ranade S, Weyer AD, Dubin AE, Baba Y, Qiu Z, Petrus M, Miyamoto T, Reddy K, Lumpkin EA, et al. 2014. Piezo2 is required for Merkel-cell mechanotransduction. Nature. 509(7502):622-626.

Yamada T, Nakamura K, Iwaku M, Fusayama T. 1983. The extent of the odontoblast process in normal and carious human dentin. J Dent Res. 62(7):798802 . 